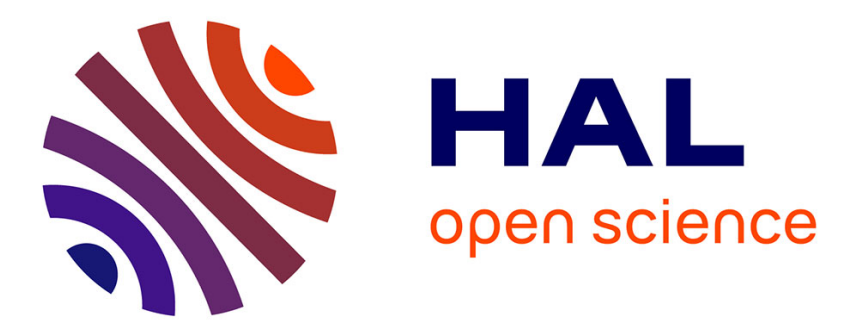

\title{
Efficacy of ST1968 (namitecan) on a topotecan-resistant squamous cell carcinoma
}

Valentina Zuco, Rosanna Supino, Enrica Favini, Monica Tortoreto, Raffaella

Cincinelli, Anna Cleta Croce, Federica Bucci, Claudio Pisano, Franco Zunino

\section{- To cite this version:}

Valentina Zuco, Rosanna Supino, Enrica Favini, Monica Tortoreto, Raffaella Cincinelli, et al.. Efficacy of ST1968 (namitecan) on a topotecan-resistant squamous cell carcinoma. Biochemical Pharmacology, 2009, 79 (4), pp.535. 10.1016/j.bcp.2009.09.012 . hal-00544815

\section{HAL Id: hal-00544815 \\ https://hal.science/hal-00544815}

Submitted on 9 Dec 2010

HAL is a multi-disciplinary open access archive for the deposit and dissemination of scientific research documents, whether they are published or not. The documents may come from teaching and research institutions in France or abroad, or from public or private research centers.
L'archive ouverte pluridisciplinaire HAL, est destinée au dépôt et à la diffusion de documents scientifiques de niveau recherche, publiés ou non, émanant des établissements d'enseignement et de recherche français ou étrangers, des laboratoires publics ou privés. 


\section{Accepted Manuscript}

Title: Efficacy of ST1968 (namitecan) on a topotecan-resistant squamous cell carcinoma

Authors: Valentina Zuco, Rosanna Supino, Enrica Favini, Monica Tortoreto, Raffaella Cincinelli, Anna Cleta Croce, Federica Bucci, Claudio Pisano, Franco Zunino

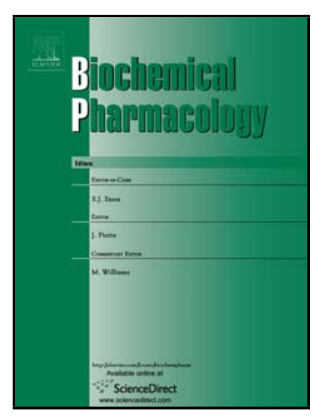

PII: S0006-2952(09)00766-7

DOI: doi:10.1016/j.bcp.2009.09.012

Reference: BCP 10326

To appear in: $\quad B C P$

Received date: $\quad$ 17-7-2009

Revised date: $\quad$ 7-9-2009

Accepted date: $\quad$ 10-9-2009

Please cite this article as: Zuco V, Supino R, Favini E, Tortoreto M, Cincinelli R, Croce AC, Bucci F, Pisano C, Zunino F, Efficacy of ST1968 (namitecan) on a topotecan-resistant squamous cell carcinoma, Biochemical Pharmacology (2008), doi:10.1016/j.bcp.2009.09.012

This is a PDF file of an unedited manuscript that has been accepted for publication. As a service to our customers we are providing this early version of the manuscript. The manuscript will undergo copyediting, typesetting, and review of the resulting proof before it is published in its final form. Please note that during the production process errors may be discovered which could affect the content, and all legal disclaimers that apply to the journal pertain. 
1

2

3

\title{
Efficacy of ST1968 (namitecan) on a topotecan-resistant squamous cell carcinoma
}

\author{
Valentina Zuco ${ }^{\mathrm{a}}$, Rosanna Supino ${ }^{\mathrm{a}}$, Enrica Favini ${ }^{\mathrm{a}}$, Monica Tortoreto ${ }^{\mathrm{a}}$, Raffaella Cincinelli ${ }^{\mathrm{a}}$, \\ Anna Cleta Croce ${ }^{\mathrm{b}}$, Federica Bucci ${ }^{\mathrm{c}}$, Claudio Pisano ${ }^{\mathrm{c}}$ and Franco Zunino ${ }^{\mathrm{a} *}$
}

${ }^{a}$ Fondazione IRCCS Istituto Nazionale Tumori, 20133 Milan, Italy; ${ }^{b}$ Consiglio Nazionale delle Ricerche, Pavia, Italy; 'Sigma-Tau, 00044 Pomezia (Rome), Italy.

\section{Category: Antibiotics and chemotherapeutics}

\author{
*Corresponding author. Fondazione IRCCS Istituto Nazionale Tumori, Via Venezian 1, 20133 \\ Milan, Italy. Tel. +39-0223902267, fax +39-0223902692. E-mail address: \\ franco.zunino@istitutotumori.mi.it
}




\section{Abstract}

ST1968 (namitecan), a novel 7-modified hydrophilic camptothecin, was found to be effective against tumor models relatively resistant to topotecan and irinotecan. Based on this observation, this study was designed to investigate the cellular and antitumor effects of ST1968 in a subline of A431, squamous cell carcinoma, selected for resistance to topotecan (A431/TPT). This model was characterized by a slow growth rate, associated with down-regulation of EGFR and topoisomerase I. In contrast to other camptothecins (SN38 and gimatecan), ST1968 was able to overcome almost completely the resistance at cellular level. The cellular pharmacokinetics indicated a comparable accumulation and retention of ST1968 in sensitive and resistant cells, in spite of expression of the efflux transporter, P-glycoprotein, in resistant cells. The uptake and retention of topotecan was dramatically reduced in both tumor cell lines, but more evident in the resistant one. In contrast to topotecan, ST1968 retained an outstanding efficacy in vivo against the resistant tumor (A431/TPT). The results are consistent with the interpretation that ST1968 was able to overcome the most relevant mechanisms associated with the development of topotecan resistance (i.e., slow proliferation and target downregulation) owing to its peculiar pharmacokinetic behaviour.

Key words: camptothecin, ST1968, topotecan, drug resistance 


\section{Introduction}

Camptothecins are among the most effective antitumor agents that stabilize the covalent binding of topoisomerase I to DNA during the enzyme catalytic process forming a reversibile ternary complex [1-3]. During DNA synthesis the collision of the replication forks with the cleavable complex (topo I-DNA) converts the single-strand breaks into irreversible lethal doublestrand breaks [1,4]. On the basis of this mechanism and of the reversibility of the cleavable complex, prolonged intracellular accumulation and stability of the ternary complex are required to exploit the S-phase-specific activity and therapeutic efficacy of camptothecins [4]. We have reported that the presence of highly lipophilic substituents at position 7 provides favourable features, in terms of cellular pharmacokinetics, lactone stability and ternary complex stabilization [5-7]. However, the ability to stabilize the cleavable complex is a favourable feature of several derivatives of the 7-oxyiminomethyl series [8]. In particular, the hydrophilic derivative namitecan (Fig. 1) exhibit an excellent activity, superior to that of irinotecan and topotecan (TPT), against several tumor models, including slowly growing tumors $[9,10]$. To better understand the basis of the peculiar profile of ST1968, we have performed a comparative study of ST1968 and topotecan in a squamous cell carcinoma model selected for resistance to topotecan (A431/TPT). The results support the ability of ST1968 to partially overcome the acquired resistance of A431/TPT cells, likely reflecting an improved uptake and persistent intracellular accumulation which allow efficient target inhibition and antitumor efficacy in spite of reduced proliferation rate and reduced topoisomerase I expression. 


\section{Materials and methods}

\subsection{Cell lines and culture conditions}

The human squamous cell carcinoma A431 and its subline selected for resistance to topotecan (A431/TPT), were maintained in RPMI 1640 (Lonza, Group Ltd, Switzerland) supplemented with $10 \%$ FBS (Gibco ${ }^{\circledR}$ Invitrogen, Segrate, Italy). The A431/TPT cell line was selected by growing A431 cells for 4 months in culture medium containing $0.05 \mu \mathrm{M}$ topotecan (Hycamtin, GlaxoSmithKline). A431/TPT cells were subcultured in the presence of the drug every two split.

\subsection{Drugs and Antibodies}

ST1968 was synthesized as previously described [5]. For in vitro studies, ST1968, gimatecan and SN-38 were dissolved in dimethylsulfoxide. Topotecan was dissolved in sterile distilled water. For in vivo studies, ST1968 was dissolved in sodium lactate buffer (50 mM) adjusted to $\mathrm{pH} 4.0$ with addition of hydrochloric acid. Topotecan was dissolved in sterile distilled water. The drugs were administered by a slow i.v. injection in a volume of $10 \mathrm{~mL} / \mathrm{kg}$ body weight.

The following antibodies were used: anti-EGFR (Upstate Biotecnologies, Lake Placid, NY); antip-glicoprotein (MDR1) and -actin (Sigma, St. Louis, MO), anti-BCRP (Alexis Biochemicals, Lausen, Switzerland), anti-MRP-1 and anti-V-ATPase D (Santa Cruz Biotecnology, Santa Cruz, CA); anti-DNA topoisomerase I (BD Bioscence, San Josè, CA).

\subsection{Cell sensitivity studies}

Cell sensitivity to the drugs was determined by growth inhibition assay. Cells were seeded in duplicate into twelve-well plates and exposed to the drugs for 1 hour. Adherent cells were 
trypsinized 72 hours after treatment and counted by a cell counter (Beckam Coulter Inc., Fullerton, CA). $\mathrm{IC}_{50}$ values, determined from dose-response curves, were defined as the drug concentrations producing $50 \%$ inhibition of cell growth. The reported values represent the mean \pm standard deviation (SD) derived from at least three independent experiments.

\subsection{Determination of apoptosis}

Apoptosis was determined by TUNEL assay 72 hours after treatment. Cells were fixed in $4 \%$ paraformaldehyde (45 min), washed and resuspended in ice-cold PBS. The "in situ cell death detection" kit (Roche, Germany) was used according to the manufacturer's instructions, and samples were analyzed by flow cytometry (FACScan, Becton Dickinson, Franklin Lakes, NJ).

\subsection{Cellular pharmacokinetics}

Cells were seeded in six-well plates at a subclonfluent density (around $100.000 \mathrm{cells} / \mathrm{cm}^{2}$ ). After 24 hours, samples, in triplicate, were washed with serum-free medium and exposed for 30 min., 1 hour or 2 hours to the drug in serum-free medium. Treated cells were washed three times with PBS to remove the extracellular drug and, for retention studies, maintained in a drug-free medium for further 5 hours. At different times, washed cells were resuspended in $150 \mu \mathrm{l}$ of PBS and subjected to 5 cycles of freezing in liquid nitrogen and thawing in thermostated bath at $37^{\circ} \mathrm{C}$. Each cycle lasted $5 \mathrm{~min}$. Cell lysates were frozen at $-20^{\circ} \mathrm{C}$. Samples were processed by adding cold $0.1 \%$ acetic acid/methanol (1:5, v:v). Analysis was performed using the 32Karat Software (Beckam-coulter) with a fluorescence detector (Jasco or Shimadzu) fixed at $370 \mathrm{~nm}$ as excitation wavelength and $510 \mathrm{~nm}$ as emission wavelength. ST1968 and topotecan were eluted on a Discovery HS F5 column (5 mm, 100x4.6 mm, Supelco) kept at room temperature in isocratic condition (0.1 M acetic acid, 0.1\% TEA:CAN, 75:25, v:v) with a flow rate of $1 \mathrm{ml} / \mathrm{min}$. 


\subsection{Intracellular drug distribution}

Subconfluent cells, grown on coverslips, were treated with ST1968 or topotecan $10 \mu \mathrm{g} / \mathrm{ml}$ for up to 2 hours. Fluorescence images were acquired at different times by means of an Argus VIM 100 processor digital system (Hamamatsu Photonics Deutschland GmbH, Herrsching am Ammersee, Germany), using an high sensitive ISIT camera (Hamamatsu C2400-09) coupled to a Leica (Wetzlar, Germany) fluorescence microscope. The images were digitally stored on a magnetic mass memory support and processed by means of the Hamamatzu Argus 100 control program. For drug localization study, images under excitation at $366 \mathrm{~nm}(366 \mathrm{~nm}$ interference filter; $\mathrm{T} \%=$ 40) were acquired at wavelengths $>440 \mathrm{~nm}$ by means of a $11000 \mathrm{v} 3$ filter combination set (Chroma Technology Corp, Rockingham, VT). The emission spectrum of topotecan was in the range of 450-600 nm with a maximum at $520 \mathrm{~nm}$. The emission spectrum of ST1968 (430-550 nm) exhibited a maximum at $480 \mathrm{~nm}$.

LysoTracker Red DND-99 (LTR) (Invitrogen) was used for lysosomal staining. Sequential images were recorded from the same field at wavelengths $>440 \mathrm{~nm}$ (covering the emission region of ST1968, in the $440-540 \mathrm{~nm}$ range, and of LTR in the $580-630 \mathrm{~nm}$ range), and $>570 \mathrm{~nm}$ (selective for LTR signal). Images were also recorded from cells incubated with LTR alone, to determine the contribution of the dye signal to the images recorded at wavelengths $>440 \mathrm{~nm}$. An image analysis was performed on each couple of images, and ratio values (F.I.> 440/F.I.> $570 \mathrm{~nm}$ ) were calculated pixel by pixel on the lysosomal areas. The comparison of the ratio values from the cells incubated with LTR alone and from those incubated with both ST1968 and LTR allowed the determination of the contribution of ST1968 signal to that of LTR in the lysosomes.

\subsection{Western blot analysis}

Cells were rinsed twice with PBS, added with $0.1 \mathrm{mM}$ sodium orthovanadate and lysed in hot 


\subsection{Assessment of lysosomal membrane permeability}

The lysosomal acidification was determined by incubating cells with $5 \mu \mathrm{M}$ acridine orange (Sigma-Aldrich, St. Louis, MO) for $17 \mathrm{~min}$ at $37^{\circ} \mathrm{C}$. FL-3 fluorescence was measured by flow cytometry (FACScan). The same samples were examined by a fluorescence microscope (Leica, Wetzlar, Germany).

\subsection{Antitumour activity in vivo}

All experiments were carried out using female athymic Swiss nude mice, 6-8 weeks-old (Charles River, Calco, Italy). Mice were maintained in laminar flow rooms keeping temperature and humidity constant. Mice had free access to food and water. Experiments were approved by the Ethics Committee for Animal Experimentation of the Fondazione IRCCS Istituto Nazionale dei Tumori of Milan according to institutional guidelines and to the UK Coordinating Committee on Cancer Research Guidelines [12].

Exponentially growing tumor cells $\left(10^{7}\right.$ cells/mouse $)$ were s.c. injected into mice flank. Tumor lines were achieved by serial s.c. passages of fragments (about $2 \times 2 \times 6 \mathrm{~mm}$ ) from growing tumors into healthy mice, as previously described [13]. Groups of 5 to 8 mice bearing s.c. tumors implanted in one or both flanks were employed. Tumor fragments were implanted on day 0 and 


\section{Results}

\subsection{Pattern of cellular response to selected camptothecins}

The A431/TPT cell subline, selected for resistance to topotecan, exhibited an increased doubling time as compared with the parental line ( $28 \mathrm{vs} 23 \mathrm{~h}$ in resistant and parental cells, respectively). The slow proliferation of resistant cells was reflected in a reduced in vivo growth rate, because the doubling time of resistant subline was substantially increased as compared with the parental line (5.8 vs 3.5 days). Table 1 shows the pattern of cross-resistance to topotecan, $\mathrm{SN}-38$ (the active metabolite of irinotecan), gimatecan and ST1968, a novel hydrophil camptothecin. The comparison of antiproliferative effects of camptothecins following $1 \mathrm{~h}$ exposure indicated a significant degree of resistance of A431/TPT to the selecting agent (TPT), SN38 and gimatecan, but only a marginal resistance to ST1968. 


\subsection{Biochemical features of the resistant subline}

Since the squamous cell carcinoma cells A431 are known to be characterized by overexpression of EGF receptor (EGFR), we determined the expression level of the protein in topotecan-resistant cells. As shown in Fig. 3, the EGFR expression was markedly reduced in A431/TPT cells as compared to the parental cells. This change could be consistent with the reduced rate of proliferation. In an attempt to explore the molecular basis of the topotecan resistance, we examined the expression of the intracellular target, topoisomerase I, and of $\mathrm{ABC}$ transporters which potentially could contribute to the resistant phenotype. A431/TPT cells revealed a marked reduction of topoisomerase I expression (Fig. 3). An analysis of the expression of proteins usually associated with multidrug resistance (Pgp, BCRP, MRP4 and V-ATPase) indicated upregulation of only Pgp (MDR1) and V-ATPase (Fig. 4A) in topotecan-resistant cells. The concomitant overexpression of the vacuolar ATP-ase has been already reported in multidrug resistant cells $[14,15]$. Since the vacuolar-type $\mathrm{H}^{+}$-ATPase (V-ATPase) is responsible for acidification of eukaryotic intracellular organelles, the lysosomal acidification was investigated by staining with acridine orange, a lysosomotropic agent which accumulates in acidic organelles 


\subsection{Cellular pharmacokinetics}

Drug uptake was studied after $30 \mathrm{~min}, 1$ and 2 hours of drug exposure, and drug retention was determined following $5 \mathrm{~h}$-incubation in a drug-free medium (Fig. 5). In A431 cells treated with 1 $\mu \mathrm{M}$, the intracellular accumulation of ST1968 was time-dependent and reached a plateau at around $2 \mathrm{~h}$-exposure [10]. The pattern of intracellular accumulation in the resistant subline was similar, but the intracellular content of ST1968 was appreciably higher than that of the parental line. The analysis of the intracellular content following $5 \mathrm{~h}$-incubation in drug-free medium indicated a substantial retention of the camptothecin in both cell lines (about $15-25 \%$ of the drug content at the end of the exposure). This behaviour, in spite of upregulation of the Pgp in the resistant cells, suggested that ST1968 was a poor substrate for this efflux transporter. At the same concentration $(1 \mu \mathrm{M})$ the intracellular accumulation of topotecan was markedly lower in either A431 and A431/TPT cells. An intracellular topotecan content comparable to that of ST1968 required exposure to $10 \mu \mathrm{M}$. Under these conditions, the plateau of drug uptake was reached at 30 min-exposure. In contrast to A431 cells, a consistent reduction of intracellular accumulation was observed in A431/TPT by increasing the exposure time. In both cell lines, the topotecan retention following removal of extracellular drug was negligible, thus suggesting a fast drug efflux.

A study of intracellular drug distribution was performed by fluorescence imaging analysis at the single cell level (Fig. 6). Both ST1968 and topotecan showed a prevailing cytoplasmic localization of the fluorescence signal, with darker areas corresponding to the nuclei, in the two 


\subsection{Antitumor activity studies}

The study of antitumor activity of topotecan and ST1968 was performed in tumor xenografts obtained by s.c. inoculation of A431 and topotecan-resistant cells in athymic nude mice. For this comparative study, we used the same treatment schedule (q4dx4, i.v. administration and optimal doses of each drug, $10 \mathrm{mg} / \mathrm{kg}$ and $25 \mathrm{mg} / \mathrm{kg}$, for topotecan and ST1968, respectively), i.e., well tolerated doses which caused acceptable body weight loss $(\leq 10 \%)$ and no toxic deaths. Under these conditions, the activity of ST1968 was substantially higher than that of topotecan in both models (Fig. 7). Topotecan caused a partial inhibition of tumor growth in A431 tumor (maximum tumor growth inhibition, around 75\%), but a marginal effect in A431/TPT, thus reflecting the 


\section{Discussion}

A number of problems limit the therapeutic efficacy of camptothecins and could account for the clinical resistance and low efficacy in several tumor types. As with other clinically effective agents, the clinical resistance to camptothecins may be a multifactorial phenomenon likely involving cellular, pharmacological and tumor-specific factors [18,19]. On the basis of the peculiar features of the camptothecin structure (i.e., opening of the lactone ring) and of the mechanism of action (i.e., conversion of the single-strand breaks in irreversible double-strand breaks during S-phase), prolonged drug exposure is a critical requisite to overcome the shortcoming related to reversibility of the cleavable complex. This is a critical aspect of the clinical use of camptothecins considering the slow growth of tumors in the clinical setting as compared to the preclinical tumor models.

The role of intracellular drug accumulation and retention as a determinant of cellular sensitivity and tumor responsivity is supported by the observations reported in our study performed on a topotecan-resistant subline of the A431 tumor. This variant selected following continuous exposure to topotecan was characterized by i) a reduced proliferation rate both in vitro and in vivo, ii) a decreased expression of topoisomerase I and iii) upregulation of MDR1. Although several factors have been implicated in the mechanisms of cellular resistance to camptothecins [18], the alterations associated with the development of resistance to topotecan 
and the pattern of cross-resistance to other camptothecins in our model may be relevant as determinants of clinical resistance, because the features of A431/TPT cells are expected to influence cellular sensitivity on the basis of the recognized mechanism of action of camptothecins.

The novel hydrophilic camptothecin of the 7-oxyiminomethyl series ST1968 exhibited ability to overcome, at least in part, the mechanisms implicated in the development of resistance in A431/TPT. In contrast to a substantial resistance to topotecan, ST1968 retained a good antitumor efficacy in vivo, in spite of the slow growth of the tumor. The cytotoxic and proapoptotic effects of ST1968 in topotecan-resistant cells were comparable to those observed in parental cells in spite of the marked reduction of topoisomerase I protein levels. A plausible explanation for the efficacy of ST1968 against this model could be its peculiar cellular pharmacokinetics characterized by a marked intracellular accumulation and retention and a peculiar subcellular distribution. This behaviour, already observed in other squamous cell carcinoma systems, is expected to ensure prolonged drug effects as suggested by the persistence of DNA damage [10]. It is conceivable that the persistence of intracellular drug may compensate the effects of the alterations which were likely responsible for the development of resistance to topotecan. Indeed, an unexpected finding of this study was a similar cellular pharmacokinetics of ST1968 in resistant and parental cells, in spite of overexpression of the transport system, Pgp, in the resistant subline. This finding suggested that ST1968 was a poor substrate for Pgp (MDR1), as already found for other derivatives of the 7-oxyiminomethyl series [20]. However, taking into account the concomitant expression of the vacuolar-ATPase in resistant cells, it is conceivable that both v-ATPase and Pgp are implicated in drug subcellular distribution, because the drug localization in cytoplasmic organelles (presumably lysosomes) was more fast and marked in the resistant subline. The acidic environment of the lysosomes may favour the stabilization of the 
lactone form of the drug and lysosomal localization could represent a store allowing intracellular release of the active drug [17]. Topotecan exhibited a different subcellular localization. However the implications of the different behaviour of the two camptothecin at subcellular level remain uncertain.

The dramatic reduction of uptake and retention of topotecan by A431 was consistent with reduced cytotoxic potency in vitro and limited antitumor efficacy. The defects in cellular accumulation of topotecan were enhanced in the resistant subline which also exhibited a reduced responsiveness in vivo (maximum TV inhibition, 30 and 75\% in A431/TPT and A431 tumor, respectively). In a cellular context characterized by low level of topoisomerase expression and a slow proliferation, it is conceivable that the intracellular concentration of topotecan was inadequate to cause sufficient lethal lesions. In contrast the favourable pharmacokinetic behaviour of ST1968 might overcome the resistance mediated by these changes, as a consequence of drug persistence inside the cell. If this interpretation is correct, the peculiar features of ST1968 may have relevant therapeutic implications in clinical development of the novel camptothecin, because the slow growth of human tumor in patients and the reversibility of drug effects are critical limitations of the efficacy of conventional camptothecins [4].

Our study indicating the efficacy of ST1968 against topotecan-resistant tumors provide further support to the therapeutic interest of the novel camptothecin. Additional advantages of ST1968 over conventional camptothecins are the improvement of the therapeutic index and efficacy in a wide range of well-tolerated doses [9]. In spite of its cytotoxic potency (higher than that of topotecan, Table 1), ST1968 was well tolerated in vivo at doses substantially higher than those of topotecan $(25-30 \mathrm{mg} / \mathrm{kg}$ vs $10-12 \mathrm{mg} / \mathrm{kg})$. A favourable pharmacokinetics characterized by a prolonged retention in tumor tissue as compared with normal tissue may account for the improvement of the therapeutic index reflecting an increased tumor selectivity of the drug 
(unpublished data). The molecular basis of the improved pharmacological profile of ST1968 remains to be defined. We have reported that ST1968, characterized by the presence of a free amino group, induces a markedly stable ternary complex [8]. Thus the molecular and pharmacokinetic features could contribute to improve antitumor efficacy and selectivity of the novel camptothecin.

\section{Acknowledgements}

This work was partially supported by the Associazione Italiana per la Ricerca sul Cancro, Milan, and by the Ministero della Salute, Rome, Italy.

\section{Disclosure of Potential Conflicts of interest}

Claudio Pisano and Federica Bucci are employees of Sigma-Tau. The other authors disclosed no potential conflicts of interest. 


\section{References}

1. Pommier Y. Topoisomerase I inhibitors: camptothecins and beyond. Nature Rev 2006; 6:789802.

2. Thomas CJ, Rahier NJ, Hecht SM. Camptothecin: current perspectives. Bioorg Med Chem 2004; 12:1585-604.

3. Teicher BA. Next generation topoisomerase I inhibitors: rationale and biomarker strategies. Biochem Pharmacol 2008; 75:1262-71.

4. Beretta GL, Zunino F. Relevance of extracellular and intracellular interactions of camptothecins as determinants of antitumor activity. Biochem Pharmacol 2007; 74:1437-44.

5. Dallavalle S, Delsoldato T, Ferrari A, Merlini L, Penco S, Carenini N, et al. Novel 7substituted camptothecins with potent antitumor activity. J Med Chem 2000; 43:3963-9.

6. De Cesare M, Pratesi G, Perego P, Carenini N, Tinelli S, Merli L, et al. Potent antitumor activity and improved pharmacological profile of ST1481, a novel 7-substituted camptothecin. Cancer Res 2001; 61:7189-95.

7. Dallavalle S, Ferrari A, Biasotti B, Merlini L, Penco S, Gallo G, et al. Novel 7oxyiminomethyl derivatives of camptothecin with potent in vitro and in vivo antitumor activity. J Med Chem 2001; 44:3264-74.

8. De Cesare M, Beretta GL, Tinelli S, Benedetti V, Pratesi G, Penco S, et al. Preclinical efficacy of ST1976, a novel camptothecin analog of 7-oxyiminomethyl series. Biochem. Pharmacol 2007; 73:656-64.

9. Pisano C, De Cesare M, Beretta GL, Zuco V, Pratesi G, Penco S, et al. Preclinical profile of antitumor activity of a novel hydrophilic camptothecin, ST1968. Mol Cancer Ther 2008; 
7:2051-9.

10. Pisano C, Zuco V, De Cesare M, Benedetti V, Vesci L, Foderà R, et al. Intracellular accumulation and DNA damage persistence as determinant of human squamous cell carcinoma hypersensitivity to the novel camptothecin ST1968. Eur J Cancer 2008; 44:133240.

11. Zuco V, Zanchi C, Cassinelli G, Lanzi C, Supino R, Pisano C, et al. Induction of apoptosis and stress response in ovarian carcinoma cell lines treated with ST1926, an atipica retinoid. Cell Deat Diff. 2004; 11:280-9.

12. Workman P, Twentyman P, Balkwill F, Balmain A, Chaplin D, Double J, et al. United Kingdom Coordinating Committee on Cancer Research (UKCCCR) guidelines for the welfare of animals in experimental neoplasia (second edition). Br J Cancer 1998; 77:1-10.

13. Polizzi D, Pratesi G, Tortoreto M, Supino R, Riva A, Bombardelli E. A novel taxane with improbe tolerability and therapeutic activity in a panel of human tumor xenograft. Cancer Res 1999; 59:1036-40.

14. Ouar Z, Bens M, Vignes C, Paulais M, Pringel C, Fleury J, et al. Inhibitors of vacuolar H+ATPase impair the preferential accumulation of daunomycin in lysosomes and reverse the resistance to anthracyclines in drug-resistant renal epithelial cells. Biochem J 2003; 370:18593.

15. Petrangolini G, Supino R, Pratesi G, Dal Bo L, Tortoreto M, Croce AC, et al. Effect of a novel vacuolar-H+-ATPase inhibitor on cell and tumor response to camptothecins. J Pharmacol Exp Ther 2006; 318:939-36.

16. Antunes F, Cadenas E, Brunk UT. Apoptosis induced by exposure to a low steady-state concentration of $\mathrm{H}_{2} \mathrm{O}_{2}$ is a consequence of lisosoma rupture. Biochem $\mathrm{J}$ 2001; 356:549-55.

17. Croce AC, Bottiroli G, Supino R, Favini E, Zuco V, Zunino F. Subcellular localization of the 
camptothecin analogues, topotecan and gimatecan. Biochem Pharmacol 2004; 67:1035-45.

18. Beretta GL, Perego P, Zunino F. Mechanisms of cellular resistance to camptothecins. Curr Med Chem 2006; 13:3291-3305.

19. Rasheed ZA, Rubin EH. Mechanisms of resistance to topoisomerase I-targeting drugs. Oncogene 2003; 22:7296-04.

20. Marchetti S, Oostendorp RL, Pluim D, van Eijndhoven M, van Tellingen O, Schinkel AH, et al. In vitro transport of gimatecan (7-t-butoxyiminomethylcamptothecin) by breast cancer resistance protein, P-glycoprotein, and multidrug resistance protein 2. Mol Cancer Ther 2007; 6:3307-13. 


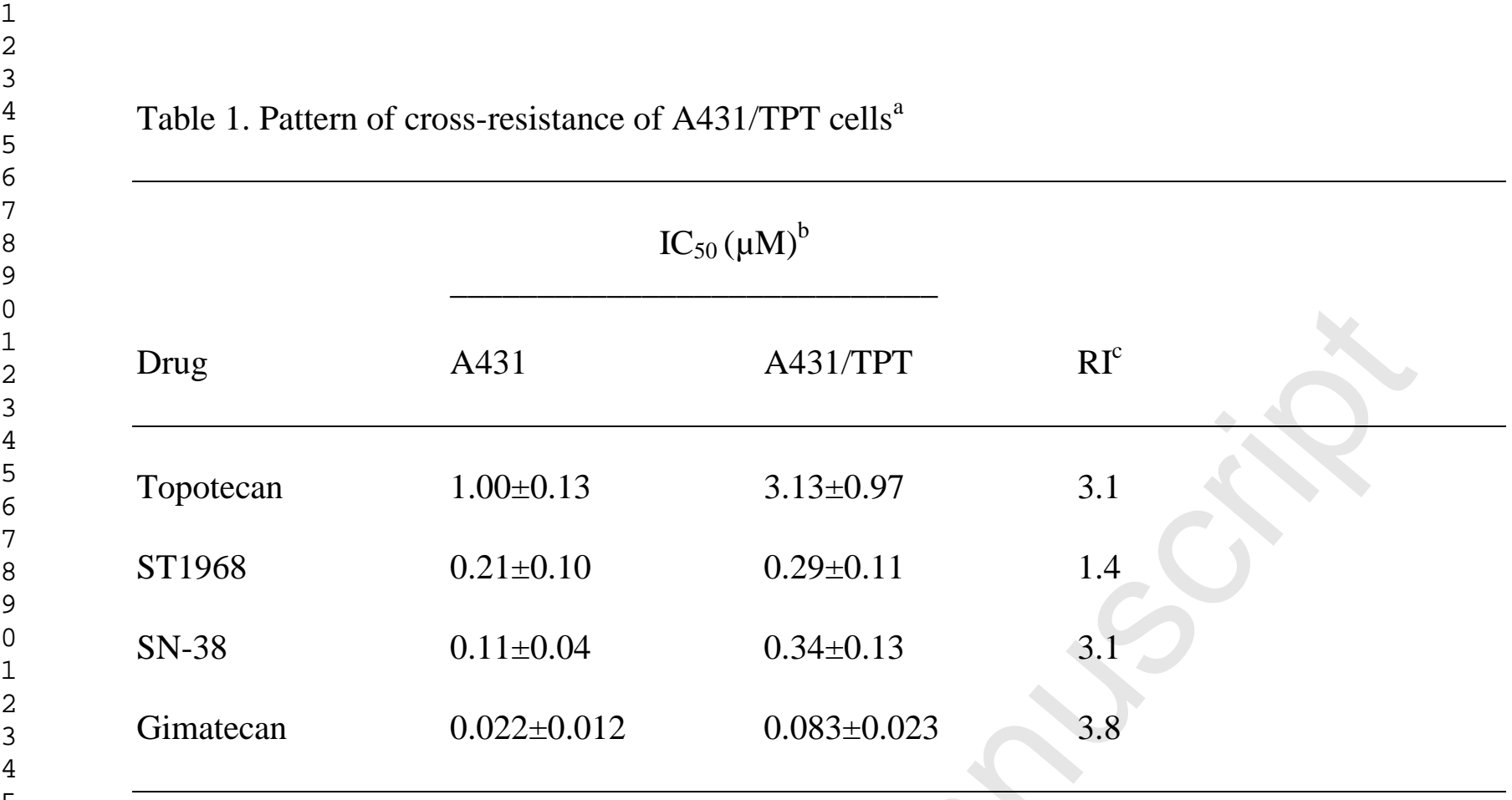

${ }^{\mathrm{a} D r u g}$ sensitivity was measured by growth inhibition assay after $1 \mathrm{~h}$ exposure.

${ }^{\mathrm{b}} \mathrm{IC}_{50}$, drug concentration causing a $50 \%$ decrease in cell growth.

${ }^{\mathrm{c}} \mathrm{RI}$, resistance index, ratio between the $\mathrm{IC}_{50}$ of topotecan-resistant and-sensitive cells. 


\section{Legends to Figures}

Figure 1. Chemical structure of ST1968.

Figure 2. Apoptosis induced by equitoxic concentrations of ST1968 and topotecan (TPT). Cells were exposed 1 hour to $2.3 \mu \mathrm{M}$ ST1968 corresponding to the $\mathrm{IC}_{80}$ value in both cell lines or to TPT 4 or $10 \mu \mathrm{M}$ corresponding to the $\mathrm{IC}_{80}$ value in A431 and A431/TPT, respectively. Apoptosis was detected by TUNEL assay and determined by FACS analysis.

Figure 3. Expression of EGFR and DNA topoisomerase I in A431 and A431/TPT cells. Protein expression was analyzed on whole cell lysates by Western blotting with the specific antibodies. Actin is shown as control for protein loading.

Figure 4. Expression of ABC transporters. (A) MDR, V-ATPase-D, BCRP and MRP-4 expression was analyzed on whole-cell lysates by Western blotting with the specific antibodies. $\beta$-tubulin is shown as control for protein loading. (B) Acridine orange staining used as marker of lysosomal acidification. Cells were incubated with $5 \mu \mathrm{M}$ acridine orange for $17 \mathrm{~min}$ at $37^{\circ} \mathrm{C}$ and intracellular red fluorescence was measured by flow cytometry. Grey profile:A431; white profile: A431/TPT. (C) acridine orange staining as by fluorescence microscopy.

Figure 5. Cellular pharmacokinetics of ST1968 and topotecan (TPT). Drug uptake was determined after $0.5,1$ or $2 \mathrm{~h}$-exposure to $1 \mu \mathrm{M}$. In the case of topotecan, intracellular drug 
content was measured also after exposure to $10 \mu \mathrm{M}$. Drug retention was determined after drug removal and 5 h-incubation in drug-free medium.

Figure 6. Intracellular drug distribution. Fluorescence images of A431 and A431/TPT cells incubated with $10 \mu \mathrm{M}$ ST1968 (A) or topotecan (B) for the indicated times were acquired by means of a processor digital system using a high-sensitivity ISIT camera coupled to a fluorescence microscope under excitation at $366 \mathrm{~nm}$ and emission > $440 \mathrm{~nm}$. Pictures were adjusted to comparable gray levels to allow an easier comparison of the fluorescence patterns. (C) Lysosomal localization of ST1968. A431/TPT cells were exposed to ST1968 (10 $\mu \mathrm{M})$ and to LysoTracker Red (LTR) $(1 \mu \mathrm{M})$ for $1 \mathrm{~h}$. Fluorescence images were acquired at wavelength $>440$ nm (ST1968 + LTR) and at wavelength > $570 \mathrm{~nm}(\mathrm{LTR})$ under excitation at $366 \mathrm{~nm}(366 \mathrm{~nm}$ interference filter, $\mathrm{T} \%=40$, mounted in a $110000 \mathrm{v} 3$ filter set cube provided by Chroma Technology Corp. (Rockingham, VT). The imaging analysis resulted in ratio values (F.I. > 440 / F.I. > 570) of $3.43 \pm 0.42$ for LTR alone and 5.20 \pm 0.6 for the lysosomal areas observed in cells incubated with both ST1968 and LTR.

Figure 7. Comparison of the antitumor activity of ST1968 and topotecan against A431 and A431/TPT tumor xenografts in nude mice. Drugs were delivered i.v. every fourth day for four times (q4dx4) starting when tumors were just palpable. Circle: control; Square: $10 \mathrm{mg} / \mathrm{kg}$ TPT; triangle: $25 \mathrm{mg} / \mathrm{kg} \mathrm{ST} 1968$. 


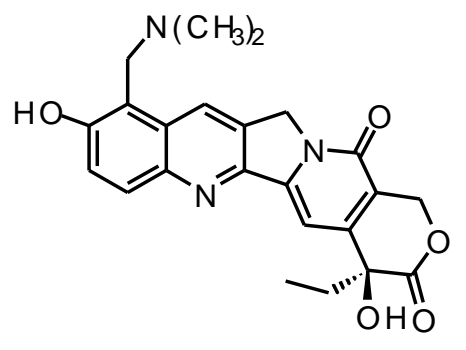

Topotecan

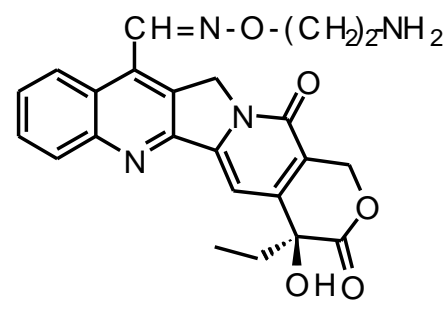

ST1968 (Namitecan)

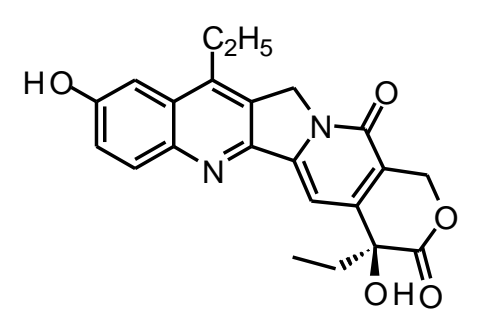

SN 38

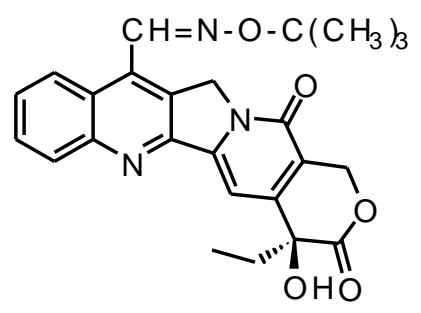

Gimatecan
Fig. 1 Zuco et al. 
A431
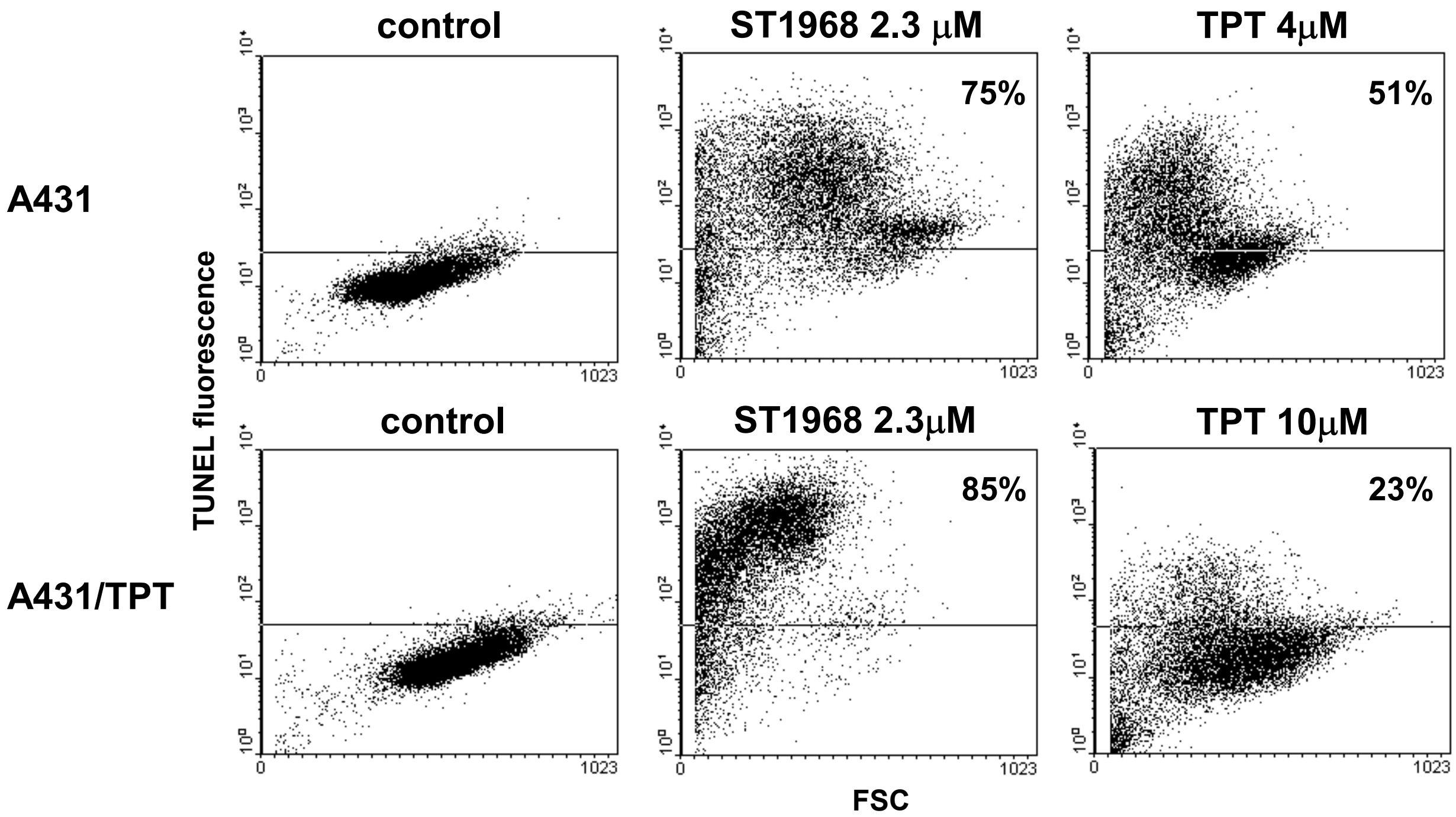

Fig. 2

Zuco et al. 


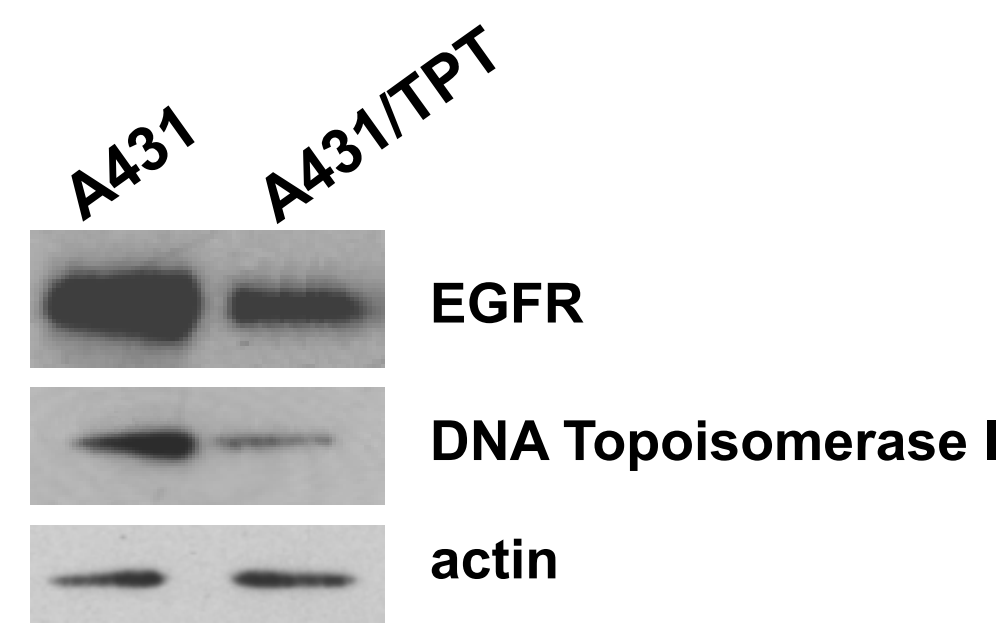

Fig. 3

Zuco et al. 
A

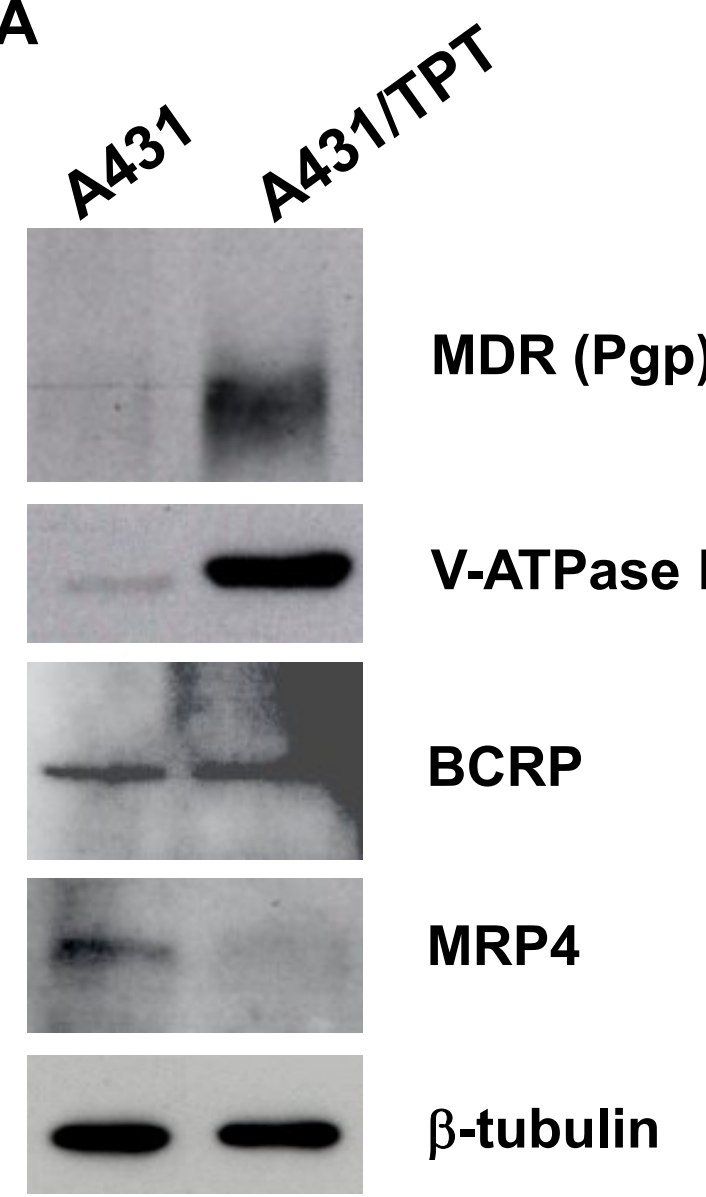

Fig. 4

Zuco et al.
B

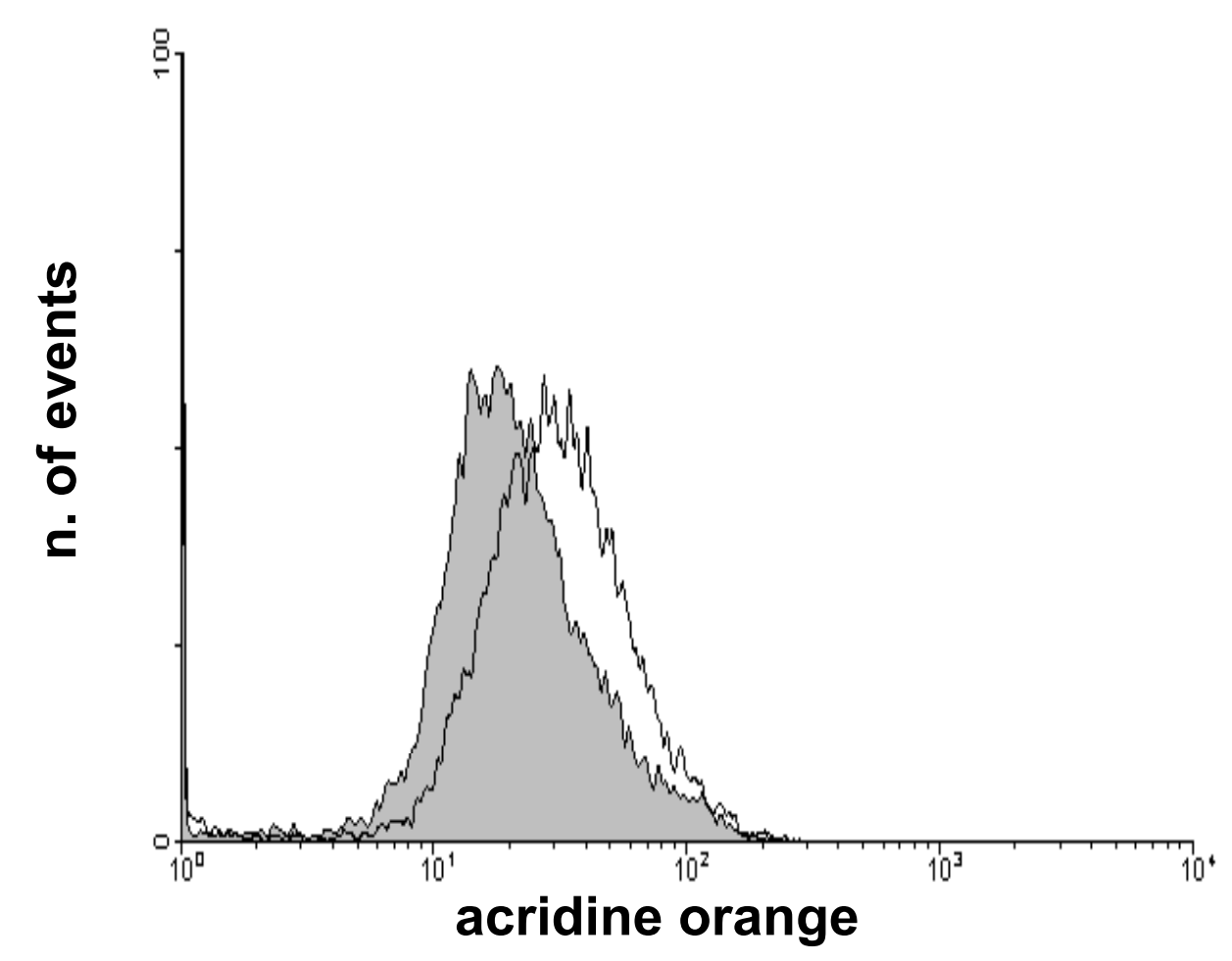

C

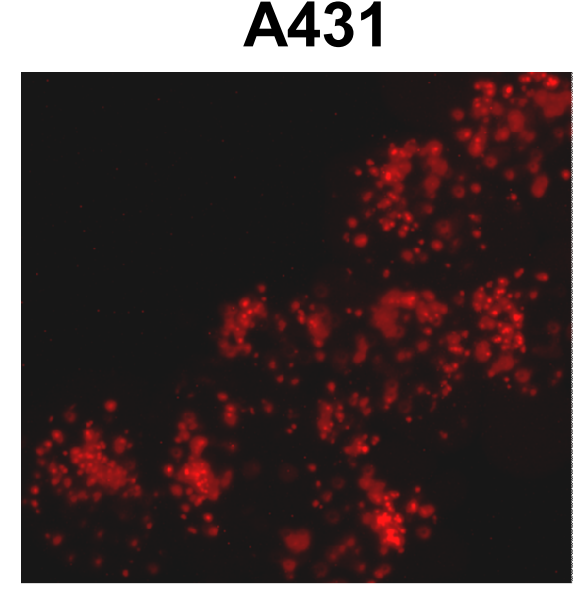

A431/TPT

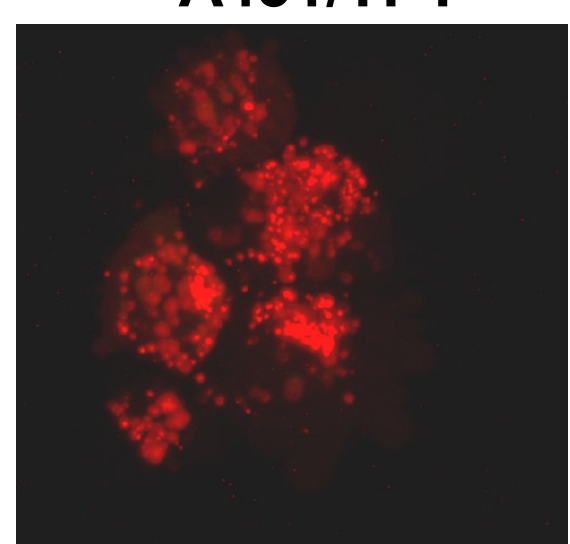




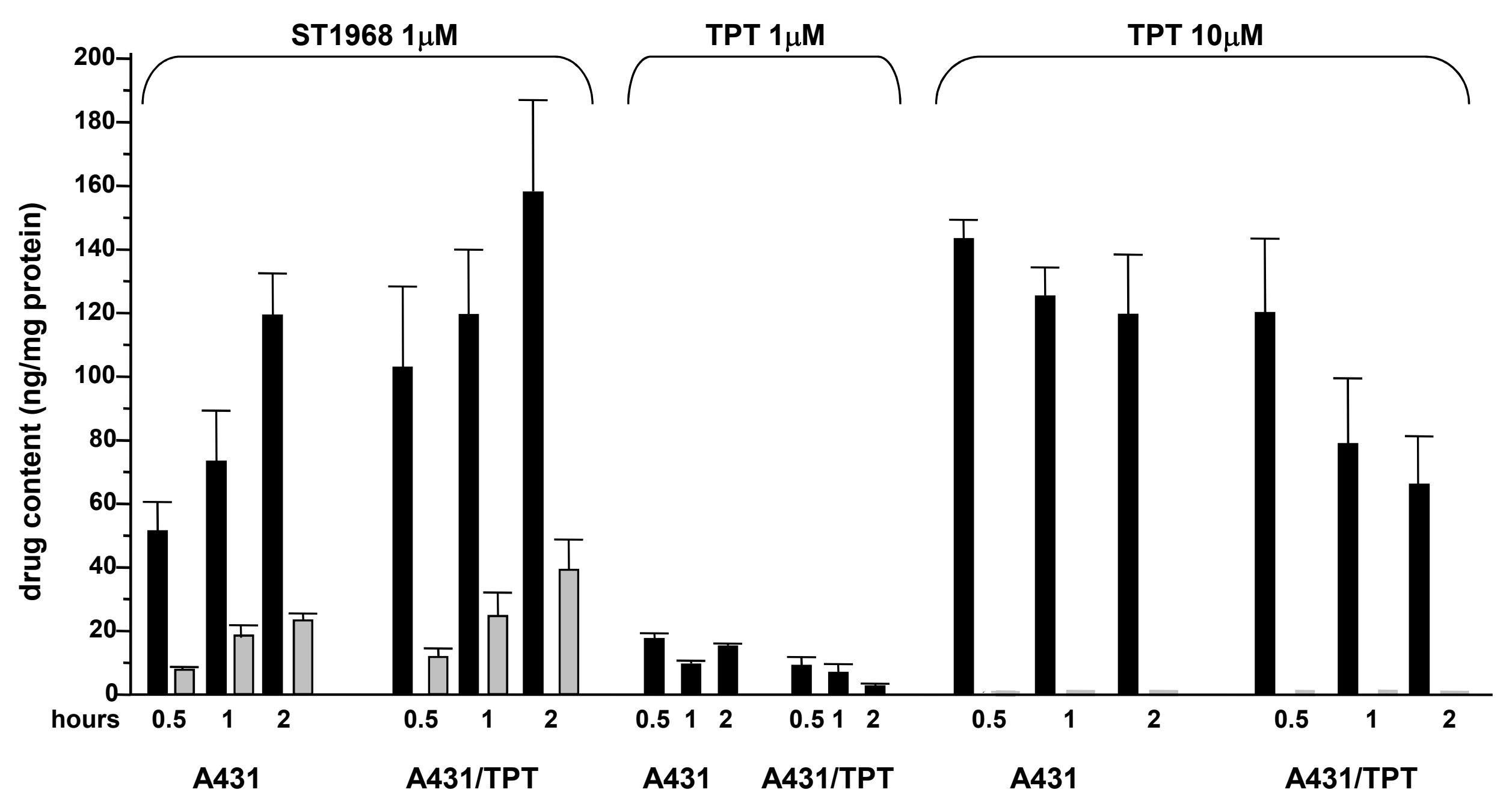

treatment end $5 \mathrm{~h}$ after drug removal

Fig. 5

Zuco et al. 
Figure 6
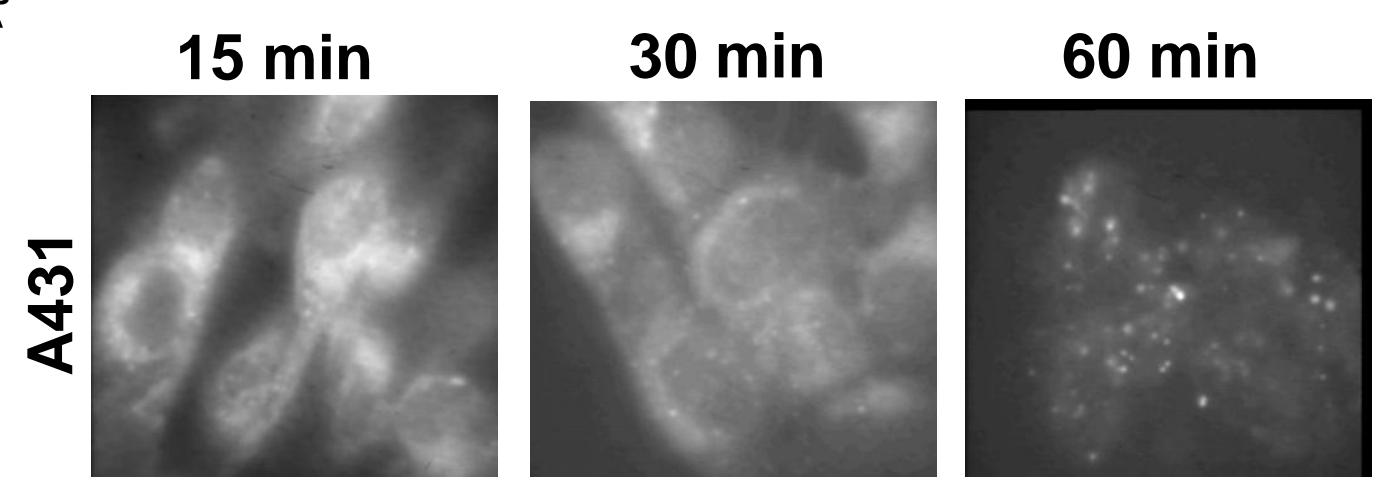

\section{$120 \mathrm{~min}$}
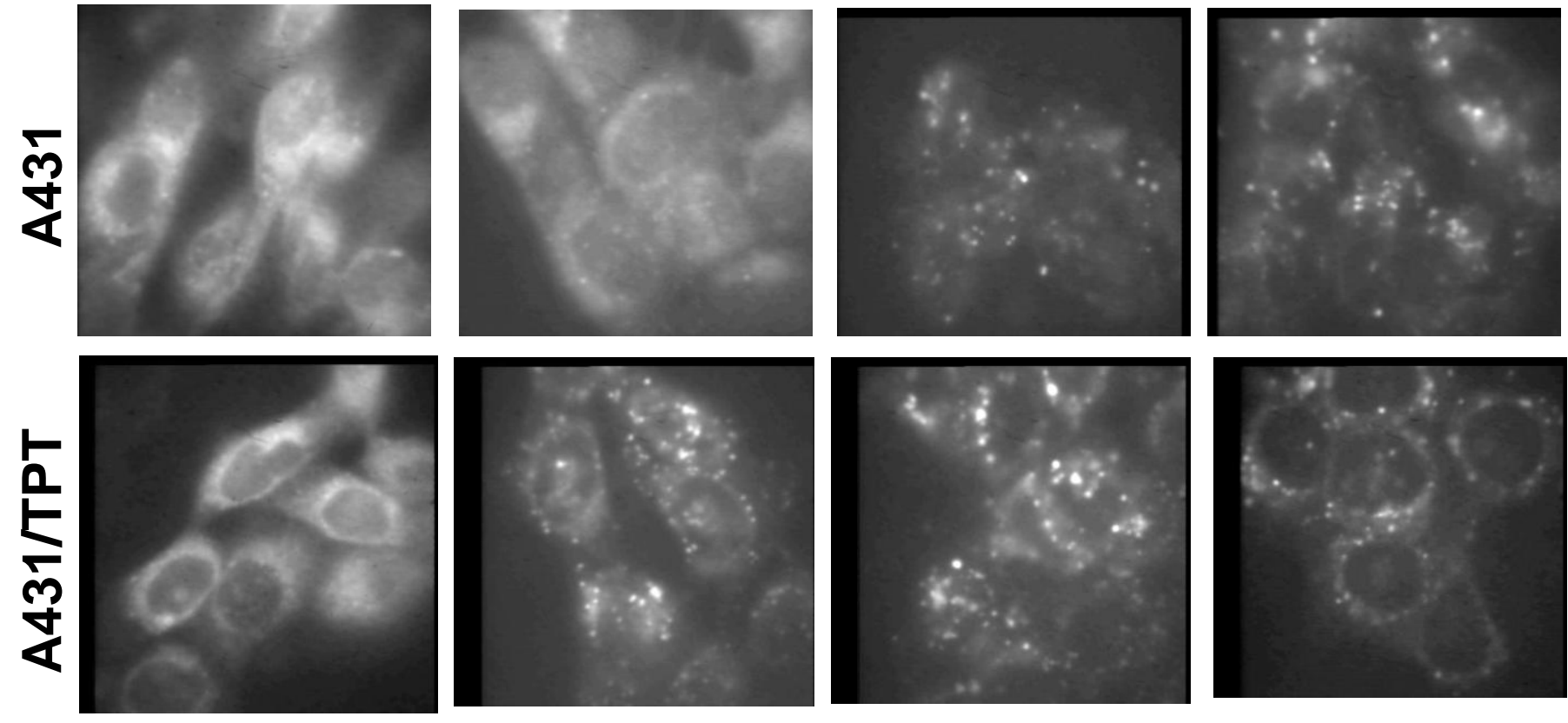

B
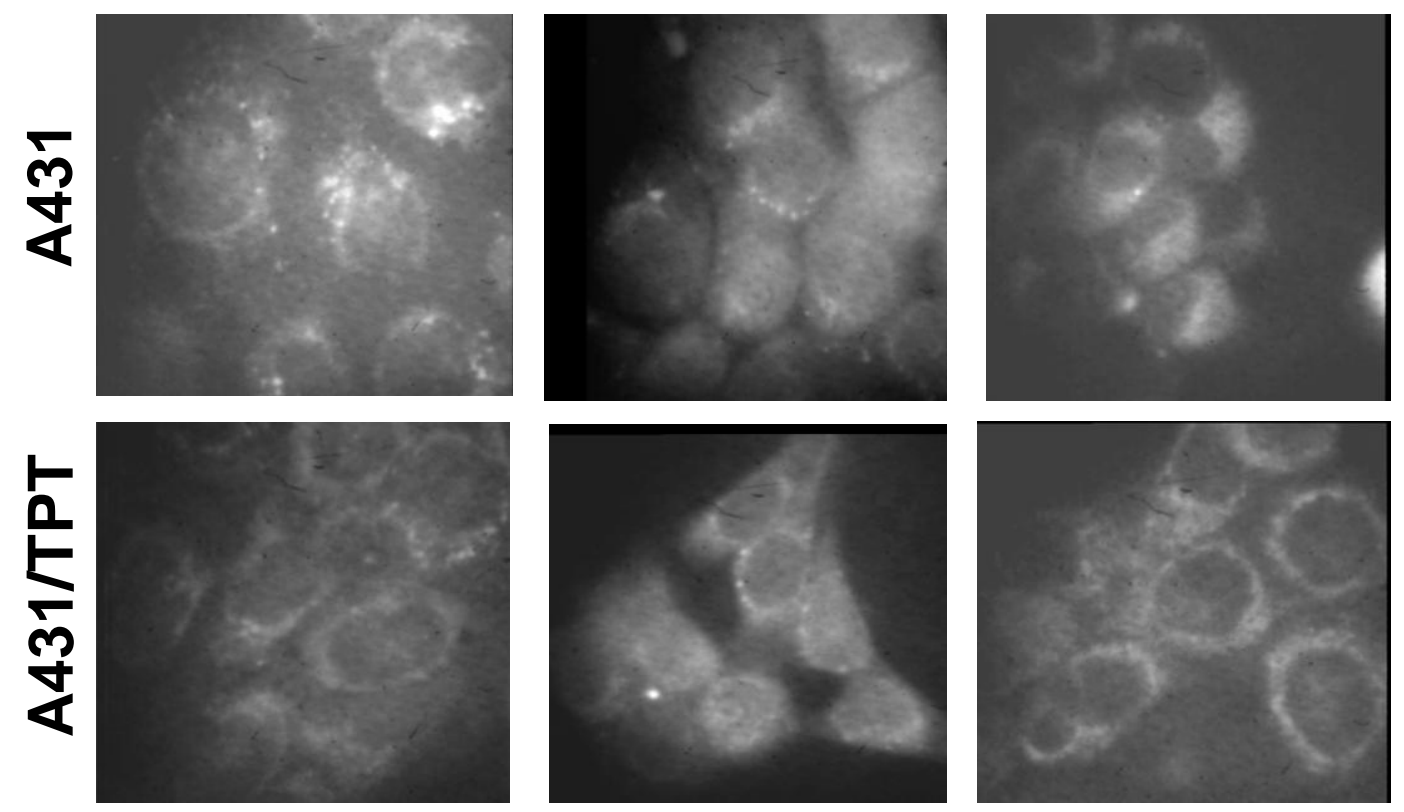

C
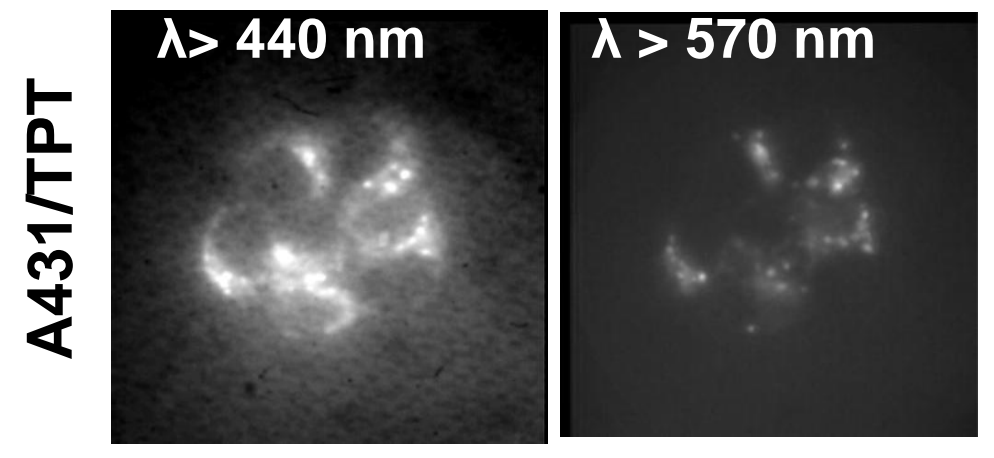

Fig. 6

Zuco et al. 

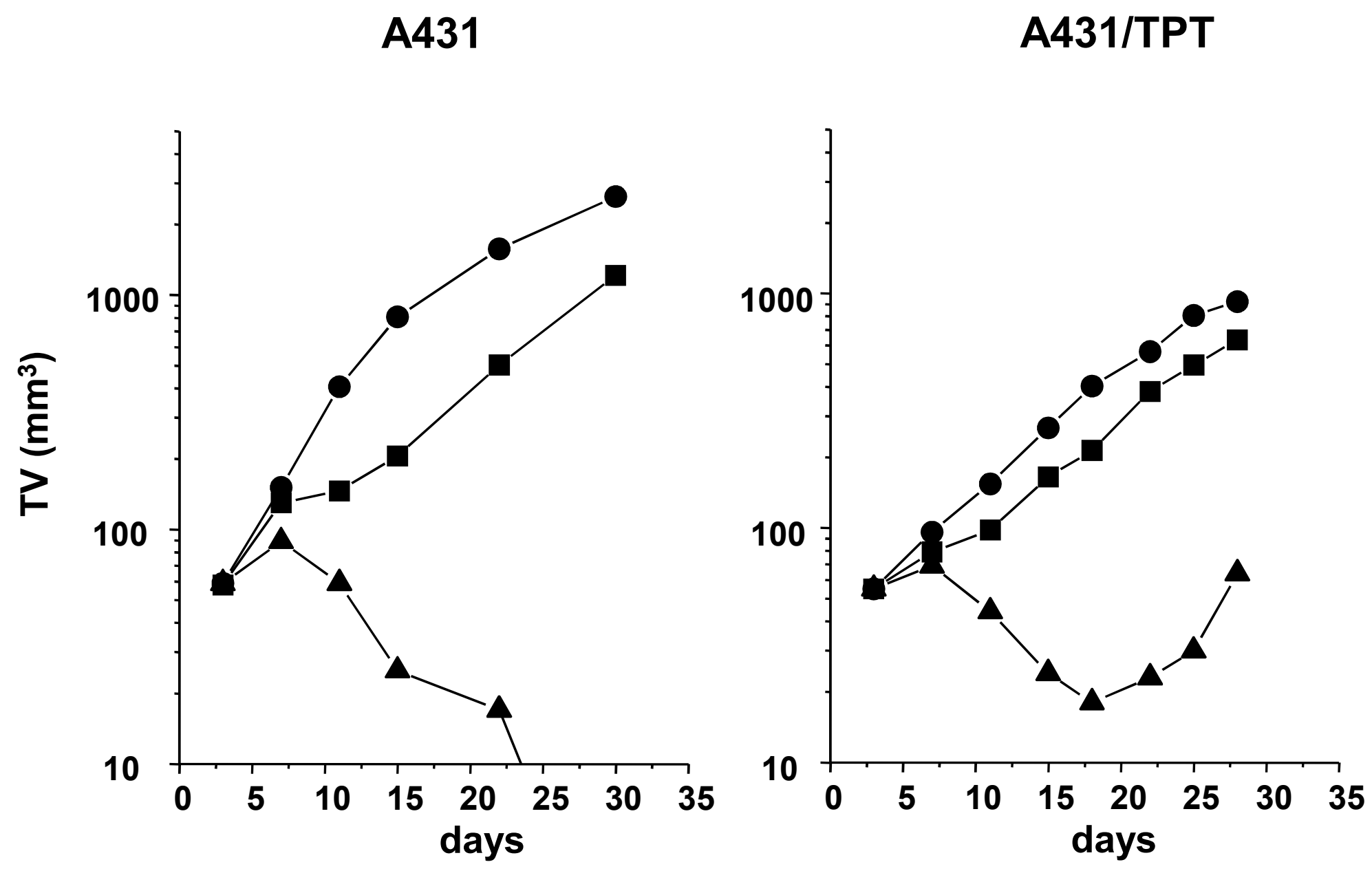

Fig. 7

Zuco et al. 